\title{
Diagnóstico casual de vitreorretinopatía exudativa familiar en 2 hermanos asintomáticos. Estudio de imagen de campo amplio y anormalidades retinianas objetivadas en el polo posterior
}

\section{Causal diagnosis of familial exudative vitreoretinopathy in 2 asymptomatic siblings. Wide-field image study of retinal abnormalities observed in the posterior pole}

\author{
Antonio Ramos-Suárez ${ }^{1 *}$, Saturnino Gismero-Moreno ${ }^{1}$, Antonio Tirado-Carmona², \\ Mercedes Lorenzo-Soto ${ }^{1}$ y Fernando García-Martín ${ }^{1}$

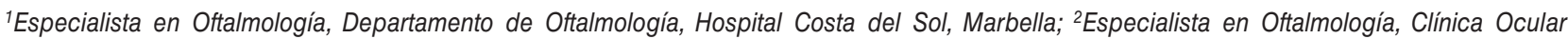 \\ Dr. Tirado, Fuengirola. Málaga, España
}

\begin{abstract}
Resumen
La vitreorretinopatía exudativa familiar es una enfermedad hereditaria rara encuadrable en el grupo de las distrofias vitreorretinianas. Se caracteriza por un desarrollo anormal de los vasos retinianos periféricos, lo que genera desde isquemia periférica leve hasta neovascularización, exudación e incluso desprendimiento de retina. Ante la dificultad de diagnóstico en casos leves y asintomáticos, recientemente se han descrito signos objetivables en el polo posterior que pueden facilitar un diagnóstico precoz. Presentamos los casos clínicos de 2 hermanos diagnosticados de vitreorretinopatía exudativa familiar casualmente durante el estudio de una uveítis en uno de ellos. La angiografía fluoresceínica de campo amplio resultó de gran ayuda para el diagnóstico y seguimiento. La mayoría de los nuevos signos descritos en polo posterior están presentes en nuestros casos. Uno de los hermanos, a pesar de encontrarse asintomático, precisó fotocoagulación con láser de la retina periférica que se realizó precozmente lo que favoreció un buen resultado anatómico y funcional.
\end{abstract}

Palabras clave: Vitreorretinopatía exudativa familiar. Distrofia. Angiografía fluoresceínica. Fotocoagulación retiniana. Retinopatía de la prematuridad.

\begin{abstract}
Familial exudative vitreoretinopathy is a rare inherited disease classified in the group of vitreoretinal dystrophies. It is characterised by the abnormal development of peripheral retinal vessels, which can provoke pathologies ranging from mild peripheral ischaemia to neovascularisation, vitreous bleeding and even retinal detachment. To overcome the difficulty of diagnosis in mild and asymptomatic cases, researchers have recently described objective signs that may be observed in the posterior pole. We present the clinical cases of 2 siblings diagnosed with familial exudative vitreoretinopathy, fortuitously, during the study of uveitis in one of them. Wide-field fluorescein angiography was of great help in the diagnosis and monitoring of this condition. Most of the new signs described in the posterior pole are present in our cases. One of the siblings,
\end{abstract}

Correspondencia:

*Antonio Ramos-Suárez

E-mail: antoniomirmarbella@ hotmail.com na de Oftalmología. Publicado por Permanyer México SA de CV. Este es un artículo Open Access bajo la licencia CC BY-NC-ND (http://creativecommons.org/licenses/by-nc-nd/4.0/).
Fecha de recepción: 22-03-2016

Fecha de aceptación: 20-05-2016

DOI: 10.1016/j.mexoft.2016.05.003
Disponible en internet: 02-07-2016 Rev Mex Oftalmol. 2018;92(1):43-49 www.rmo.com.mx 
despite being asymptomatic, required laser photocoagulation of the peripheral retina. This was performed at an early stage, which enabled good anatomical and functional results to be achieved.

Key words: Familial exudative vitreoretinopathy. Dystrophy. Fluorescein angiography. Retinal photocoagulation. Retinopathy of prematurity.

\section{Introducción}

La vitreorretinopatía exudativa familiar (VREF) es un trastorno hereditario poco frecuente descrito por Criswick y Schepens en $1969^{1}$. Como ocurre en la retinopatía del prematuro (ROP), el trastorno primario consiste en un fallo en el crecimiento de los vasos retinianos en la periferia afectando más frecuentemente a la arteria temporal. Las complicaciones secundarias de la enfermedad dependerán del grado de isquemia ${ }^{2,3}$. La herencia suele ser autosómica dominante (HAD), aunque puede ser autosómica recesiva (HAR) o ligada al $X$. También se han descrito casos sin antecedentes familiares de la enfermedad ${ }^{2}$. Presenta un alto grado de penetrancia pero expresividad variable. Sus características clínicas incluyen retina periférica avascular, neovascularización, exudación subretiniana y anormalidades en la interfase vitreorretiniana. En casos más evolucionados pueden aparecer pliegues falciformes en retina, desprendimiento traccional y hemorragias vítreas recurrentes ${ }^{2-6}$. Ambos ojos suelen afectarse aunque de forma característicamente asimétrica. Además pueden existir desde casos asintomáticos a otros severos dentro de la misma familia².

Presentamos los casos de 2 hermanos con signos de avascularidad en la retina periférica de ambos ojos.

\section{Caso clínico 1}

Niño de 12 años que consulta por miodesopsias en el ojo izquierdo (OI) sin antecedentes personales de interés. Como antecedente familiar, el padre biológico presenta una trombofilia con mutación del factor $\mathrm{V}$ de Leyden.

La máxima agudeza visual corregida era de unidad y la presión intraocular de $12 \mathrm{mmHg}$ en ambos ojos (AO).

El polo anterior era normal en el ojo derecho (OD) y presentaba un Tyndall celular de 1+ en el Ol sin otros hallazgos. La funduscopia del OD se consideró normal, mientras que en el Ol se evidenció un Tyndall vítreo celular de 1+, edema de papila con borramiento de los bordes, y leve avascularidad en la retina temporal periférica (Fig. 1).

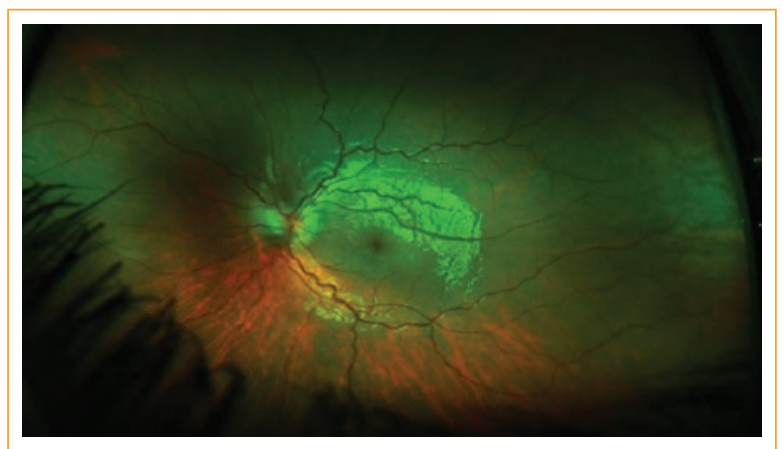

Figura 1. Retinografía de campo amplio en OI. Edema de papila con borramiento de bordes y discreta avascularidad en retina periférica temporal.

Se realizó angiografía fluoresceínica (AFG) de campo amplio que mostraba un punteado coroideo hiperfluorescente bilateral en hemirretina inferior, más marcado en el OI donde además se asociaba hiperfluorescencia papilar, todo ello compatible con un cuadro uveítico. Puso de manifiesto además cierta dilatación capilar y leve avascularidad de la retina periférica temporal e inferior (Fig. 2A y B) en ausencia de signos de vasculitis, que en ese momento no fue relacionada con una distrofia vitreorretiniana. Con estos hallazgos, el paciente fue diagnosticado de uveítis posterior y se comenzó su estudio sistémico, seguimiento y tratamiento médico.

\section{Caso clínico 2}

Niña de 9 años asintomática y hermana biológica de los mismos padres que el caso 1. Es traída a consulta por sus padres adoptivos tras el diagnóstico a su hermano biológico de uveítis posterior de etiología no filiada. Entre los antecedentes personales procede destacar el parto a las 32 semanas de gestación con $1,496 \mathrm{~g}$ de peso que no precisó oxígeno. No se objetivaron signos de ROP en la evaluación realizada por la unidad de oftalmología infantil.

La máxima agudeza visual corregida era de unidad y la presión intraocular de 14 mmHg en AO. 

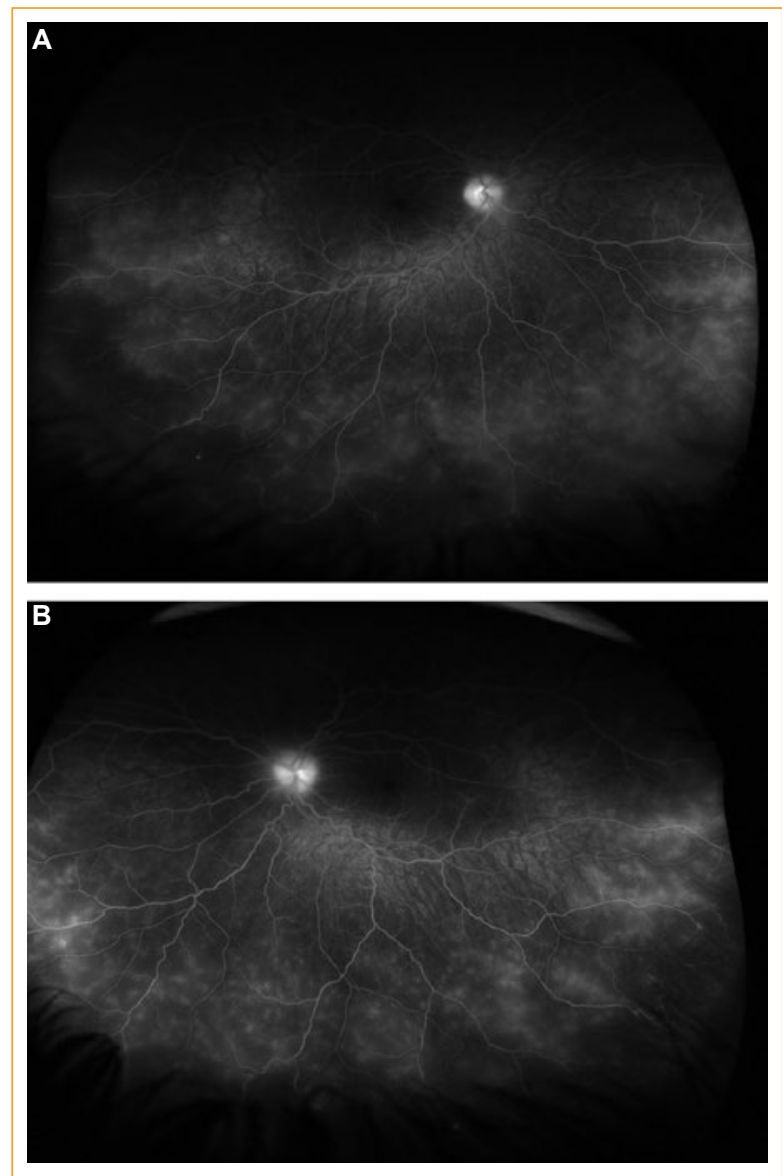

Figura 2. A y B: Angiografías de campo amplio. Hiperfluorescencia papilar y punteado coroideo hiperfluorescente más intenso en Ol y en hemirretina inferior. Leve avascularidad en retina periférica temporal e inferior.

El polo anterior era normal en AO. La funduscopia en $\mathrm{OD}$ evidenció avascularidad en retina temporal periférica. En el Ol se observó exudación en la retina periférica temporal con neovascularización y leve estiramiento vascular (Fig. 3A y B).

Se realizó AFG de campo amplio que confirmó la neovascularización periférica temporal objetivándose intenso rezume de colorante en OI. En OD presentaba leve avascularidad en retina periférica temporal (Fig. 4A y B). Dados los antecedentes, la edad de diagnóstico, los hallazgos y la asimetría en la clínica, la paciente es diagnosticada de VREF.

Se decide realizar tratamiento con fotocoagulación periférica en $A O$. A los 3 meses se realiza nueva AFG que muestra persistencia de la neovascularización en Ol (Fig. 5A y B) por lo que se procede a confluir la fotocoagulación, consiguiendo la regresión de los
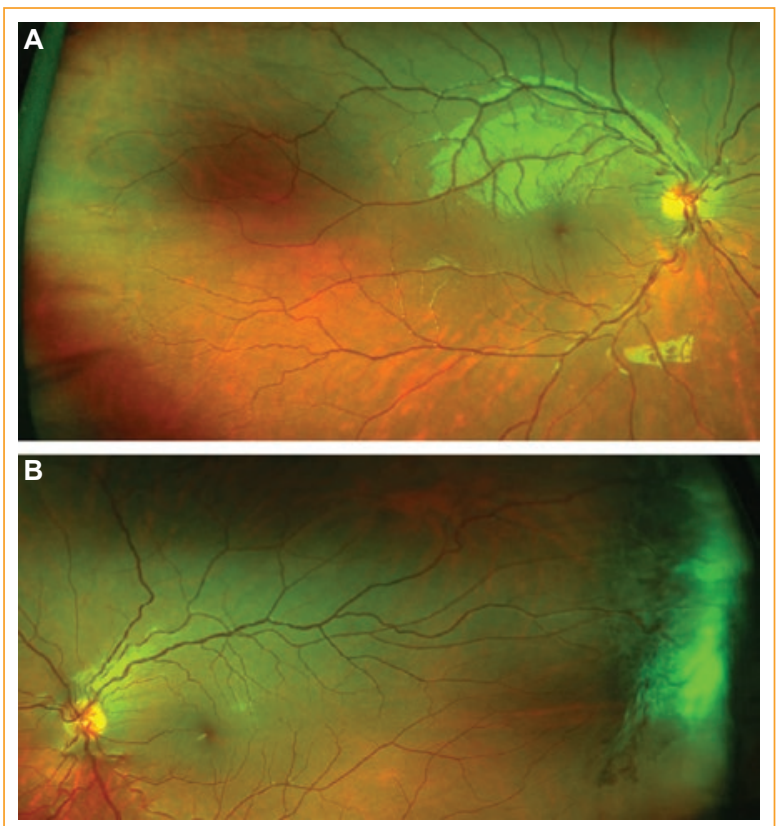

Figura 3. Retinografías de campo amplio.

A: OD: Avascularidad en periferia temporal.

B: OI: Proliferación fibrovascular con estiramiento vascular y neovascularización.

neovasos que se mantiene tras 9 meses de seguimiento (Fig. 6A y B).

En el estudio sistémico del hermano no se objetivó enfermedad asociada a la uveítis aunque precisa de $17,5 \mathrm{mg}$ de metotrexato semanales para su tratamiento.

Tras el diagnóstico de la hermana y los hallazgos descritos en la funduscopia y AFG, fue considerado un caso leve de VREF. Se decidió realizar un seguimiento periódico del paciente que permanece asintomático tras 12 meses.

\section{Discusión}

La VREF es una entidad rara en la que un fallo en la angiogénesis retiniana conduce a una vascularización incompleta en su periferia lo que origina isquemia, justificando esta los hallazgos clínicos. Suele diagnosticarse en la infancia aunque puede aparecer a cualquier $\mathrm{edad}^{7}$, a diferencia de la ROP cuyo comienzo es en la primera infancia. La presentación inicial varía desde pequeñas zonas avasculares en retina periférica hasta un desprendimiento de retina total. Su evolución es impredecible.

Existen 3 estadios clínicos ${ }^{8,9}$ :

- Estadio I: alteraciones en la interfase vitreorretiniana como blanco con o sin presión o degeneración 

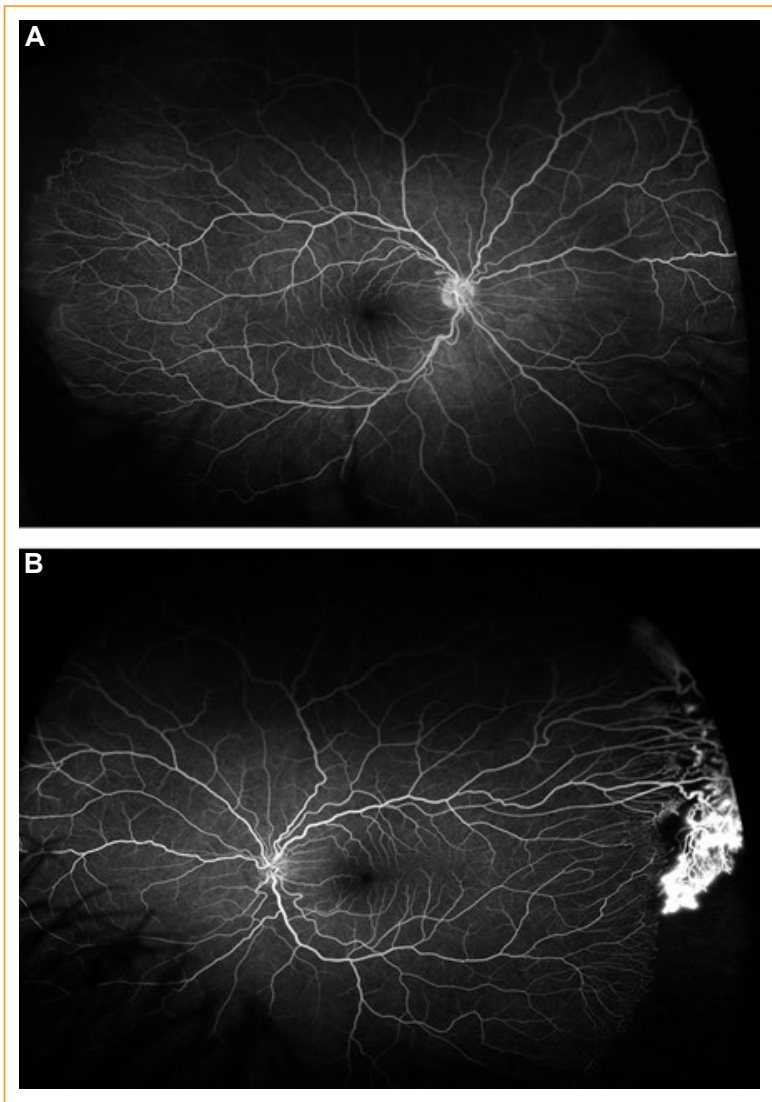

Figura 4. Angiografías de campo amplio. A: OD: Retina periférica avascular. B: OI: Retina periférica avascular y neovasos con intenso rezume de colorante.

cistoide. Zonas avasculares en retina periférica. Los pacientes no presentan sintomatología.

- Estadio II: aparece neovascularización, proliferación fibrovascular y exudación. La tracción puede originar ectopia macular.

- Estadio III: aparece desprendimiento de retina que suele ser traccional. También puede ser exudativo o regmatógeno.

El diagnóstico suele ser clínico. En el fondo de ojo podemos encontrar avascularidad a menudo de la retina periférica temporal, neovascularización en la frontera entre la retina perfundida y la avascular, exudación, fibrosis y contracción de la retina periférica, incluso desprendimiento de retina seroso o traccional. La contracción de la retina periférica puede inducir estiramiento y tracción del polo posterior, incluso ectopia macular y en casos severos la formación de pliegues retinianos falciformes. Raramente puede dar lugar a una displasia retiniana completa ${ }^{2,4}$. La AFG de campo amplio resulta de gran ayuda para el diagnóstico y el seguimiento por su capacidad de explorar la
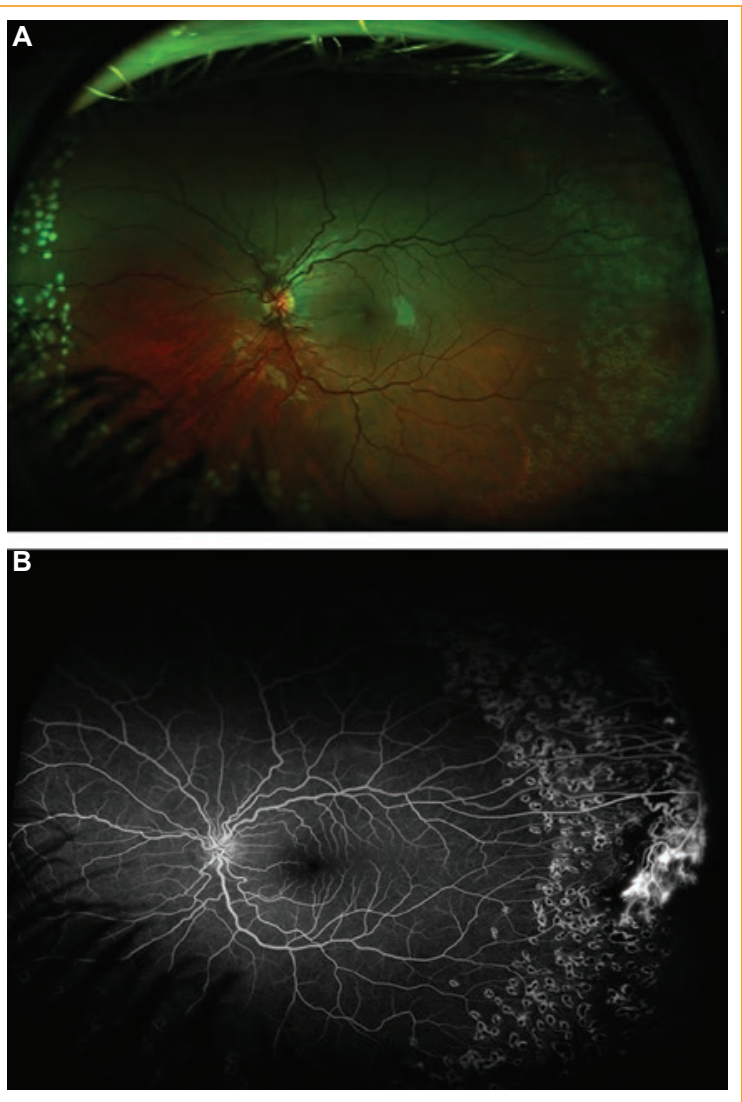

Figura 5. A y B: Retinografía y angiografía de campo amplio de 0l. Fotocoagulación periférica y persistencia de la neovascularización.

retina periférica. En ocasiones facilita observar anormalidades que podrían pasar más fácilmente desapercibidas en la funduscopia. Es una herramienta útil para diagnosticar casos familiares con fenotipos menos severos $^{3,7}$. En nuestros casos facilitó la detección de la avascularidad en la retina periférica temporal que, aunque presente, era menos evidente en la funduscopia.

Recientes estudios han descrito anomalías microestructurales en el polo posterior y alteraciones en la interfase vitreorretiniana presentes en casos asintomáticos. La tomografía de coherencia óptica de dominio espectral podría detectar la mayoría de dichas alteraciones en pacientes con VREF, convirtiéndose en una herramienta útil en el diagnóstico precoz de esta enfermedad, sin embargo se precisan más estudios al respecto ${ }^{3,6}$. En nuestros casos, las tomografías de coherencia óptica maculares fueron normales.

Yuan et al. ${ }^{4}$ describen varios hallazgos clínicos novedosos que podrían facilitar un diagnóstico precoz de los pacientes con VREF leve. Según ellos, los 

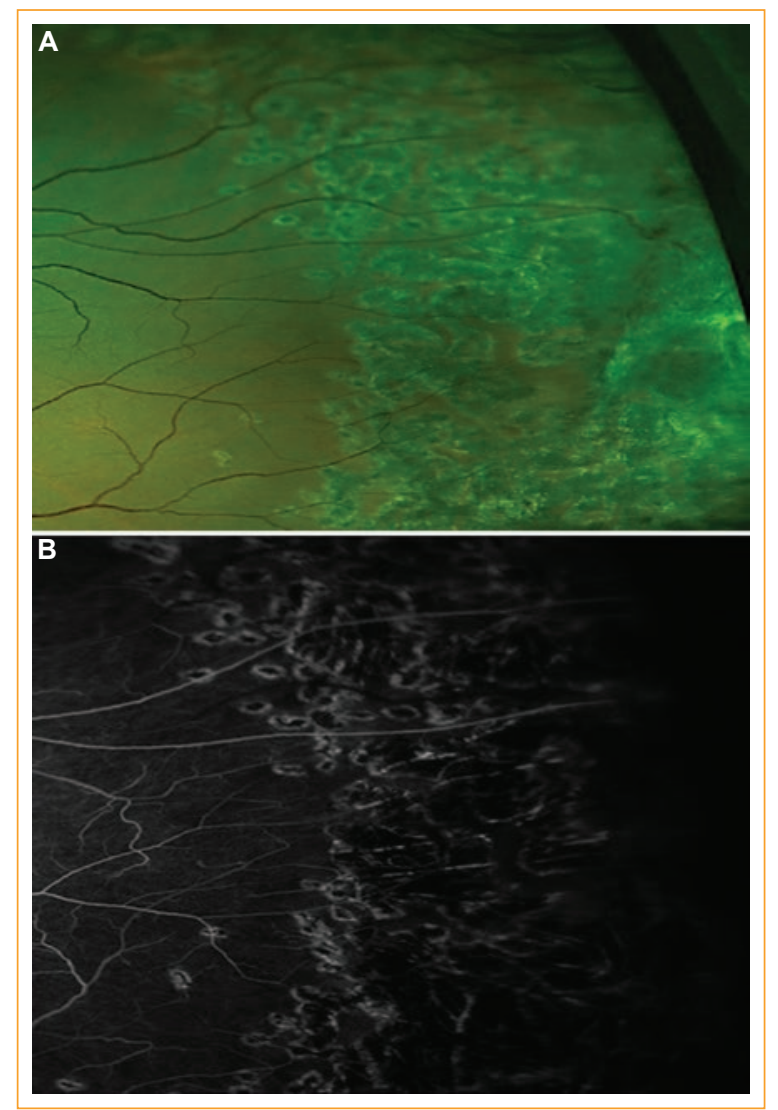

Figura 6. A y B: Retinografía y angiografía de campo amplio de Ol. Ausencia de neovasos a los 6 meses de seguimiento tras realizar una fotocoagulación más confluente.

pacientes con VREF en estadios I o II presentan varias anormalidades objetivables en el polo posterior con respecto al grupo control, como una distancia del disco óptico a la mácula (DM) significativamente mayor, y un diámetro papilar (DP) más pequeño por un diámetro horizontal reducido. EI DP es calculado como la media entre los diámetros papilares vertical y horizontal. En nuestro primer caso, el DP del OD fue de $1.78 \mathrm{~mm}$ y de $1.80 \mathrm{~mm}$ en OI. La DM fue de $4.56 \mathrm{~mm}$ en el OD y de $5 \mathrm{~mm}$ en el Ol. En nuestro segundo caso, el DP del OD fue de $2.01 \mathrm{~mm}$ y de $2.05 \mathrm{~mm}$ en el Ol. La DM fue de $5.18 \mathrm{~mm}$ en el OD y de $5.73 \mathrm{~mm}$ en el Ol. En ambos casos, la DM es similar a la obtenida por Yuan et al. en el grupo de pacientes con VREF salvo el OD del primer caso que presenta una DM normal. Sin embargo, el DP es mayor en nuestros 2 casos, siendo más llamativa la diferencia en el segundo caso, el cual presenta un DP mayor que el grupo control del trabajo de Yuan et al.
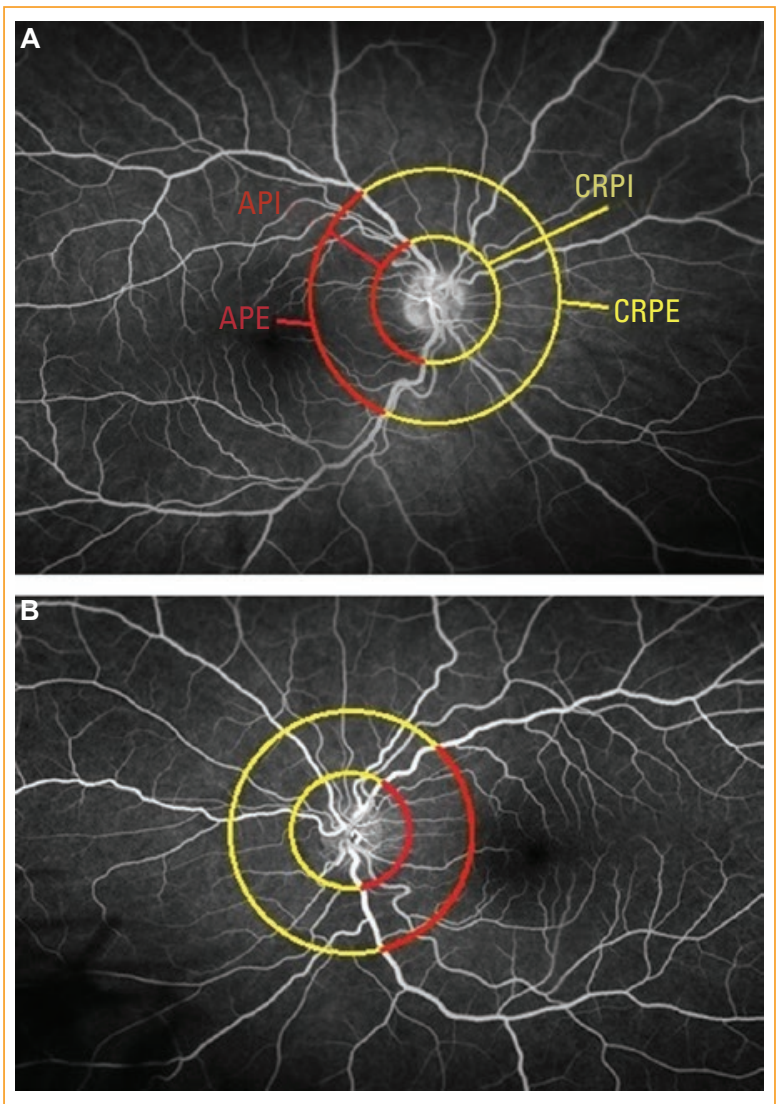

Figura 7. A y B: Angiografías del caso 2 y representación de los círculos y arcos peripapilares.

Además, describen que el número de vasos que partirían desde el disco óptico en pacientes con VREF sería mayor que en la población sana. Para el recuento de los vasos trazan 2 círculos concéntricos a la papila y 2 arcos dentro de dichos círculos. El círculo de referencia peripapilar interno (CRPI) tiene un diámetro de 2 veces el disco óptico mientras que el círculo de referencia peripapilar externo tiene un diámetro de 4 veces el disco óptico. El arco peripapilar interno sería aquella porción de CRPI comprendida entre la vena temporal superior e inferior de la retina mientras que el arco peripapilar externo sería aquella porción de círculo de referencia peripapilar externo comprendida entre la vena temporal superior e inferior de la retina (Fig. 7A y B).

En el primero de nuestros casos, con hallazgos clínicos sugestivos de VREF muy leve, el número de vasos que cruzan el CRPI es de 23 en el OD y de 17 en el Ol. El arco peripapilar interno es cruzado por 10 vasos en $\mathrm{AO}$. En nuestro segundo caso, el número de 
vasos que cruzan el CRPI es de 25 en el OD y de 23 en el Ol; y el número de vasos que cruzan el arco peripapilar interno es de 12 en el OD y de 11 en el Ol. Estos resultados son similares a los obtenidos por Yuan et al. en pacientes con VREF salvo por el número de vasos que cruzan el CPRI en el Ol en el primero de nuestros casos, que fue menor, hecho que podría deberse al ser un caso muy leve de la enfermedad.

Se han identificado 5 genes que cuando sufren mutaciones pueden producir VREF: NDP (ligado al X), FZD4 (HAD y HAR), LRP5 (HAD y HAR), TSPAN12 (HAD y HAR), y ZNF408 (HAD). Las mutaciones en estos 5 genes explican alrededor del $50 \%$ de los casos de VREF 2 . Algunos de ellos tienen un papel fundamental en la vía de señalización de la proteína par Norrin/Frizzled-4, importante para el desarrollo embrionario de una adecuada estructura tridimensional en la red vascular retiniana ${ }^{2,9}$. Las mutaciones en el gen LRP5 pueden producir también osteopenia y osteoporosis. Por ello algunos autores como Gilmour defienden que todos los pacientes diagnosticados de VREF en los que los test genéticos no estén disponibles, deben ser sometidos a una densitometría ósea. Esto permite un diagnóstico y tratamiento precoz que permitiría prevenir posibles fracturas óseas ${ }^{2}$.

Por otro lado, Shastry et al..$^{10}$ reportan el caso de una familia donde existe VREF con mutación del gen FZD4 asociado a mutación del factor $V$ Leiden sugiriendo una herencia digénica de la enfermedad en algunos casos. Sin embargo otros autores atribuyen dicha asociación al azar ${ }^{11}$. En este sentido, el padre biológico presentó una trombofilia secundaria a una mutación del factor $\mathrm{V}$ Leiden aunque no se encontraron alteraciones hematológicas en nuestros casos.

La base del tratamiento consiste en fotocoagulación láser de la retina isquémica ${ }^{12}$. Estudios recientes reflejan que la terapia intravítrea con fármacos anti-VEGF podría resultar beneficiosa ${ }^{13,14}$. El manejo en estadios iniciales es controvertido. Algunos autores recomiendan valorar cada caso de forma individual y tratar solo aquellos en los que exista alto riesgo de progresión ${ }^{15}$. Otros por el contrario apuestan por un tratamiento intensivo con láser o crioterapia de forma $\mathrm{precoz}^{8}$. El tratamiento en casos más severos con desprendimiento de retina puede requerir cirugía con cerclaje y/o vitrectomía ${ }^{12}$.

El pronóstico es muy variable como consecuencia del diferente grado de afectación en el momento del diagnóstico y de su curso impredecible. Dada la afectación severa del Ol en el segundo caso, iniciamos tratamiento con PFC periférica en AO por riesgo de progresión. Sin embargo, decidimos una actitud expectante con revisiones periódicas en nuestro primer caso. Los pacientes menores de 3 años o aquellos que comienzan con neovascularización presentan un peor pronóstico ${ }^{15}$.

El diagnóstico diferencial de esta enfermedad incluye:

- ROP: Ambas pueden presentarse con una periferia avascular, neovascularización o desprendimiento de retina. La ROP presenta un curso constante con neovascularización sobre la 37 semana de edad gestacional y desprendimiento de retina sobre la 41. No progresa ni recurre durante el resto de la infancia o vida adulta. La afectación suele bilateral y simétrica. Se asocia a prematuridad, bajo peso y empleo de oxigenoterapia tras el parto.

- Persistencia de la vasculatura fetal o vítreo primario hiperplásico persistente: Caracterizado por la presencia de membranas fibrovasculares retrolentales como consecuencia de una ausencia en la regresión de la vasculatura hialoidea. Suele ser unilateral y esporádica. Los ojos afectados suelen ser microftálmicos.

- Enfermedad de Norrie: Se asocia a mutación del gen NPD. Es extremadamente rara y simula una VREF severa salvo porque es bastante simétrica. La padecen los varones (herencia ligada al $X$ ) y puede asociar microftalmia y opacidad corneal, lo que ayuda a diferenciarla de la VREF. Con frecuencia presentan sordera progresiva y retraso mental, cuya presencia apoya el diagnóstico.

- Síndrome de osteoporosis seudoglioma: Trastorno autosómico recesivo por mutaciones del gen LRP5. Cursa con ceguera congénita o en la infancia y osteoporosis juvenil severa. Las alteraciones oculares pueden ir desde phthisis bulbi hasta afectaciones vitreorretinianas menos severas como hiperplasia, pliegues retinianos falciformes congénitos 0 exudación retiniana. Además pueden presentar baja estatura, microcefalia, laxitud ligamentosa, hipotonía y retraso mental.

- Incontinentia pigmenti: Puede cursar con avascularidad de la retina periférica y desarrollo de neovascularización durante la infancia o más tarde. Como la VREF, suele ser asimétrica. Se hereda ligada al $X$ y es una enfermedad letal intraútero en la mayoría varones, por lo que las alteraciones descritas se ven en mujeres o en los pocos casos que son varones tienen un cariotipo 47XXY, una alteración hipomórfica con menos mutaciones deletéreas 0 mosaicismo somático por mutación de una sola cromátide. Las alteraciones sistémicas son la clave 
para el diagnóstico: lesiones cutáneas neonatales (transitorias), hipoplasia dental y alopecia.

- Enfermedad de Coats: Puede ser similar clínicamente pero es esporádica, idiopática y casi siempre unilateral. Consiste en anormalidades vasculares retinianas que cursan con exudación lipídica. La mayoría se diagnostican con menos de 20 años (2/3 antes de los 10 años). El 85\% son varones. Puede cursar desde solo telangiectasias periféricas asintomáticas a desprendimiento exudativo de retina total.

- Toxocariasis: Suele ser unilateral y adquirida. En una de sus formas aparece un granuloma periférico que puede causar tracción retiniana y pliegues falciformes.

- También debe de considerarse presentaciones atípicas de retinoblastoma o retinosquisis juvenil ligada al cromosoma $X$.

\section{Conclusión}

La VREF es una entidad rara pero con capacidad de producir un severo deterioro en la visión del paciente. La forma de presentación puede ser muy variable incluso dentro de una misma familia. Estudios recientes tratan de identificar alteraciones microestructurales que estarían presentes en los fenotipos menos agresivos de la enfermedad. En este sentido, la tomografía de coherencia óptica de dominio espectral se ha empleado como herramienta útil en varios estudios, sin embargo las pruebas básicas de diagnóstico y seguimiento siguen siendo la valoración funduscópica y la angiografía. Además se han descrito alteraciones anatómicas novedosas en polo posterior que pueden facilitar el diagnóstico precoz de los casos más leves. Por otro lado, los avances en genética han permitido conocer mejor la patogenia de esta enfermedad y posibles asociaciones con otras alteraciones sistémicas como osteopenia u osteoporosis.

\section{Responsabilidades éticas}

Protección de personas y animales. Los autores declaran que para esta investigación no se han realizado experimentos en seres humanos ni en animales.
Confidencialidad de los datos. Los autores declaran que han seguido los protocolos de su centro de trabajo sobre la publicación de datos de pacientes.

Derecho a la privacidad y consentimiento informado. Los autores han obtenido el consentimiento informado de los pacientes y/o sujetos referidos en el artículo. Este documento obra en poder del autor de correspondencia.

\section{Financiamiento}

Los autores no tienen revelaciones financieras que declarar.

\section{Conflicto de intereses}

Los autores declaran no tener conflicto de intereses.

\section{Bibliografía}

1. Criswick V, Schepens C. Familial exudative vitreoretinopathy. Am J Ophthalmol. 1969;68:578-94.

2. Gilmour D. Familial exudative vitreoretinopathy and related retinopathies. Eye. 2015;29:1-14.

3. Yonekawa Y, Thomas BJ, Drenser KA, et al. Familial exudative vitreoretinopathy: Spectral-domain optical coherence tomography of the vitreoretinal interface, retina, and choroid. Ophthalmology. 2015;122(11):2270-7.

4. Yuan M, Yang Y, Yu S, et al. Posterior pole retinal abnormalities in mild asymptomatic FEVR. Invest Ophthalmol Vis Sci. 2014;56:458-63.

5. Biccas Neto L, Mesquita AS, Louro ID. Familial exudative vitreoretinopathy (FEVR) associated with infantile osteoporosis: case report. Arq Bras Oftalmol. 2009;72:257-60.

6. Shimouchi A, Takahashi A, Nagaoka T, et al. Vitreomacular interface in patients with familial exudative vitreoretinopathy. Int Ophthalmol. 2013;33:711-5.

7. Ranchod TM, Ho LY, Drenser KA, et al. Clinical presentation of familia exudative vitreoretinopathy. Ophthalmology. 2011;118(10):2070-5.

8. Piñero A, Sempere J, Nadal J, Elizalde-Montagut J. Vitreorretinopatía exudativa familiar: nuestra experiencia. Arch Soc Esp Oftalmol. 2008:83(12):703-7.

9. Miller KE, Willis MJ, McClatchey SK. A case of familial exudative vitreoretinopathy identified after genetic testing. J AAPOS. 2015;19:178-80.

10. Shastry B, Trese M. Cosegregation of two unlinked mutant alleles in some cases of autosomal dominant familial exudative vitreoretinopathy. Eur J Hum Genet. 2004;12:79-82.

11. Bottomley HM, Downey LM, Inglehearn CF, et al. Comment on 'cosegregation of two unlinked mutant alleles in some cases of autosomal dominant familial exudative vitreoretinopathy'. Eur J Hum Genet. 2006;14:6-7.

12. Pendergast SD, Trese MT. Familial exudative vitreoretinopathy: Results of surgical management. Ophthalmology. 1998;105:1015-23.

13. Quiram PA, Drenser KA, Lai MM, et al. Treatment of vascularly active familial exudative vitreoretinopathy with pegaptanib sodium (Macugen). Retina. 2008;28:S8-S12

14. Sisk RA, Berrocal AM, Albini TA, et al. Bevacizumab for the treatment of pediatric retinal and choroidal diseases. Ophthalmic Surg Lasers Imaging Retina. 2010;41:582.

15. Benson WE. Familial exudative vitreoretinopathy. Trans Am Ophthalmol Soc. 1995:93:473-521. 